\title{
Variação temporal do zooplâncton da Praia de Tramandaí, Rio Grande do Sul, com ênfase em Copepoda
}

\author{
Tatiana R. Avila ${ }^{1}$, Catarina S. Pedrozo ${ }^{2}$ \& José Guilherme F. Bersano ${ }^{3}$
}

1. Laboratório de Zooplâncton, Instituto de Oceanografia, Universidade Federal do Rio Grande. Av. Itália Km 8, Campus Carreiros, Rio Grande, RS, Brasil. (tatioceano@yahoo.com.br)

2. Laboratório de Zooplâncton, Departamento de Ecologia, Universidade Federal do Rio Grande do Sul, Av. Bento Gonçalves 9500 , prédio 43422, Caixa Postal 15007, 91540-000 Porto Alegre, RS, Brasil. (catarina@ecologia.ufrgs.br)

3. Universidade Federal do Paraná, Centro de Estudos do Mar, Av. Beira Mar s/n, Caixa Postal 50002, 83255-000 Pontal do Paraná, PR, Brasil. (bersano@ufpr.br)

\begin{abstract}
Temporal variation of the zooplankton from Tramandaí Beach, RS, southern Brazil, with emphasis on Copepoda. The temporal variability of density, species composition, biomass and diversity of the surf zone zooplankton from Tramandaí Beach, RS, Brazil, was studied between August 2005 and August 2006. Samples were taken forthnightly using a zooplankton net of $150 \mathrm{~cm}$ total length, $50 \mathrm{~cm}$ mouth diameter and $300 \mu \mathrm{m}$ nylon mesh size. Data on clorofila- $a$, wind direction, longshore current, salinity, air and water temperature were taken as well. Copepoda presented the highest species diversity, being Temora turbinata (Dana, 1849) the species with higher relative abundance and frequency of occurrence. Other Copepoda species were also numerically important, such as Acartia tonsa (Dana, 1849), Subeucalanus pileatus (Glesbrecht, 1888) and Ctenocalanus vanus (Glesbrecht, 1888). The Mysidacea Metamysidopsis elongata atlantica (Bascescu, 1968) presented frequency of $58.33 \%$ and relative abundance of $44 \%$. The peak of mesozooplanktonic biomass $\left(96 \mathrm{mg} \cdot \mathrm{m}^{-3}\right)$ was recorded in September/2005, corresponding to the maximum density value presented for this species $\left(3,535\right.$ org. $\left.\mathrm{m}^{-3}\right)$. Information about zooplankton of this region is scarce and the results of this study will provide a base for the knowledge about the biological processes occurring in the surf zone of Tramandaí Beach.
\end{abstract}

KEYWORDS. Surf zone, Tramandaí Beach, Zooplankton, Copepoda, Metamysidopsis elongata atlantica.

RESUMO. Com o objetivo de conhecer a variação temporal da composição, abundância, diversidade e biomassa do zooplâncton da zona de arrebentação da Praia de Tramandaí, Rio Grande do Sul, amostragens quinzenais foram realizadas entre agosto de 2005 e agosto de 2006. Os arrastos foram efetuados com rede cilindro-cônica com $150 \mathrm{~cm}$ de comprimento, $50 \mathrm{~cm}$ de diâmetro de boca e malha de 300 $\mu \mathrm{m}$. Dados de clorofila- $a$, direção do vento, corrente de deriva litorânea, salinidade, temperatura do ar e da água também foram obtidos. O grupo dos Copepoda foi responsável pela maior diversidade de espécies, sendo que Temora turbinata (Dana, 1849) apresentou maior abundância relativa e freqüência de ocorrência. Outras espécies de Copepoda também foram importantes numericamente como Acartia tonsa (Dana, 1849), Subeucalanus pileatus (Glesbrecht, 1888) e Ctenocalanus vanus (Glesbrecht, 1888). O Mysidacea Metamysidopsis elongata atlantica (Bascescu, 1968) apresentou frequiência de 58,33\% e abundância relativa de $44 \%$, sendo o pico de biomassa de mesozooplâncton $\left(96 \mathrm{mg} . \mathrm{m}^{-3}\right.$ ) registrado em setembro de 2005 correspondente ao máximo valor de densidade apresentado pela espécie (3.535 org. $\mathrm{m}^{-3}$ ). Informações sobre o zooplâncton desta região são muito escassos e os dados levantados servirão de base para o conhecimento dos processos biológicos que ocorrem na coluna d'água da Praia de Tramandaí.

PALAVRAS-CHAVE. Zona de arrebentação, Tramandaí, zooplâncton, Copepoda, Metamysidopsis elongata atlantica.

Praias arenosas são ecossistemas presentes nas linhas de costa de diversos locais no mundo, sendo utilizadas pelos seres humanos como fonte de renda através da pesca e para lazer e recreação. Diferenças nas morfologias da praia e nas zonas de arrebentação são facilmente identificadas, podendo ser notadas zonas largas com sequiências de quebras de onda até praias onde existe apenas uma linha de quebra, ou a ausência da mesma (McLaChlan, 1980; Brown \& McLaChlan, 1990).

Estas diferenças estão relacionadas principalmente com o grau de exposição a ondas à que está submetida determinada região. Praias mais abrigadas apresentam pouca energia e menor zona de arrebentação (praias refletivas) enquanto regiões abertas apresentam alta energia de ondas e são fortemente trabalhadas pelas mesmas. Estas regiões comumente possuem um declive de praia menos acentuado, uma zona de arrebentação larga e rasa e com grande dissipação de energia (praias dissipativas). Consequentemente apresentam intensa formação de correntes de retorno e deriva litorânea (McLachlan, 1980; Brown \& McLachlan, 1990).
A dinâmica das praias dissipativas permitem a recirculação da matéria orgânica dentro de seus limites (MCLACHLAN, 1980) através da decomposição bacteriana e do reaproveitamento dos nutrientes pelos organismos do fitoplâncton, afetando de forma positiva o crescimento do zooplâncton e de outros componentes da fauna praial. Os organismos zooplanctônicos são os principais consumidores primários dos oceanos e transferem a energia fixada pelas algas para os níveis tróficos superiores. Sendo a zona de arrebentação um local de criação para algumas espécies, principalmente teleósteos zooplanctófagos, estes organismos possuem grande importância no ecossistema como base da cadeia alimentar das larvas de peixes e crustáceos explorados comercialmente (LASIAK, 1986; BROWN \& MCLACHLAN, 1990).

O zooplâncton marinho é dominado por copépodes, sendo este o grupo com melhor descrição e maior conhecimento do Oceano Atlântico Sul (Boltovsкoy, 1999). Nas zonas de arrebentação tendem a possuir maior diversidade, dividindo com Mysidacea a abundância em termos de biomassa (BERSANO, 1994). 
As praias da região de Tramandaí, assim como toda a costa do Rio Grande do Sul, são dominadas pela ação de ondas e possuem um caráter aberto e exposto diretamente à ação de ondas de energia média a elevada (Tomazelli \& VIlLwock, 1991). A zona de arrebentação formada recebe aporte de águas continentais através dos sangradouros presentes na linha de costa e do estuário do Rio Tramandaí, considerado um berçário e regulador dos estoques pesqueiros. Este é formado pelas lagunas do Armazém, Tramandaí e canal de descarga, recebendo toda a carga hídrica procedente do sistema de lagunas e desaguando no mar pelo canal (SOARES, 2002). Além disto, o padrão de circulação das águas oceânicas na região é influenciado pela confluência de massas de origens distintas, sendo a água tropical, transportada pela Corrente do Brasil, a de maior presença no verão, e as massas de água subtropical e subantártica, transportadas pela Corrente das Malvinas, as de maior intensidade durante o inverno (СIOTTI et al., 1995; SOARES \& MÖLLER, 2001; SouZa et al., 2006).

Considerando que os organismos do zooplâncton têm sua distribuição de larga escala ligada diretamente ao movimento das massas de água, as assembléias zooplanctônicas podem ser modificadas de acordo com a origem e mistura das águas que predominam numa determinada região (Boltovskoy, 1999). Em Tramandaí estas origens são variáveis (SOARES \& MöLlER, 2001; SoARes, 2002), podendo estes organismos servir de indicadores desta amplitude de influências (BoltovsкоY, 1999). No entanto, informações sobre o zooplâncton desta região são muito escassos e em função desta carência, o presente estudo teve como objetivos estudar sua variação temporal em termos de composição, abundância, diversidade e biomassa na zona de arrebentação da Praia de Tramandaí, com ênfase em Copepoda.

\section{MATERIAL E MÉTODOS}

Coletas e análises em laboratório. Este estudo foi desenvolvido em um ponto situado na zona de arrebentação da Praia de Tramandaí (Fig. 1) próximo a plataforma de pesca do balneário $\left(30^{\circ} 00^{\prime} 17^{\prime}\right.$ 'S $50^{\circ} 07^{\prime} 45^{\prime}$ 'O), sendo coletada uma amostra quinzenalmente entre agosto de 2005 e agosto de 2006.

A cada amostragem, a temperatura da água foi medida com um termômetro de mercúrio e a salinidade com um refratômetro. Para se determinar a concentração de clorofila- $a$ no momento da amostragem, coletou-se água do mar com um recipiente plástico com volume de 11, o qual foi acondicionado no escuro em uma caixa de isopor com gelo. Em laboratório, sub-amostras entre 5 e $50 \mathrm{ml}$ (conforme a concentração de algas) foram filtradas em triplicata em filtros de $0,47 \mu \mathrm{m}$ (GF 52-C) e levadas ao congelador. A extração de pigmentos seguiu a metodologia de PARSONs et al. (1984) e por leitura em um fluorímetro (Laboratório de Fitoplâncton, FURG). A direção da corrente de deriva foi obtida com um flutuador neutro (garrafa plástica preenchida com 50\% de água do mar, amarrada a um cabo de $10 \mathrm{~m}$ de comprimento). Os dados de direção e velocidade do vento à $10 \mathrm{~m}$ de altura, foram cedidos pela estação meteorológica de Tramandaí, Epagri/Ciram
Uma rede de plâncton cilindro-cônica com malha de $300 \mu \mathrm{m}$, aro de $0,5 \mathrm{~m} \mathrm{e} 1,5 \mathrm{~m}$ de comprimento foi utilizada para a coleta do zooplâncton. Arrastos horizontais de superfície com aproximadamente 3 min de duração foram realizados dentro da zona de arrebentação numa profundidade em torno de $1,5 \mathrm{~m}$. Um fluxômetro foi acoplado à boca da rede a fim de se determinar o volume de água filtrada. Após as coletas todo o material obtido foi fixado com solução de formaldeído neutralizado com Tetraborato de Sódio em concentração final de 4\%.

A identificação a nível específico e quantificação dos organismos (ênfase em Copepoda) foram realizadas sob microscópios estereoscópico e óptico sempre que necessário, utilizando-se para isto os guias compilados por Björnberg (1981), Montú \& Goeden (1986) e BRADFORD-GRIEVE et al. (1999).

A biomassa zooplanctônica em peso seco foi determinada para alíquotas representando $10 \%$ do volume total da amostra, sendo todos os organismos presentes na alíquota separados dos detritos e pesados em dois grupos, crustáceos e os chamados "gelatinosos" (sifonóforos, hidromedusas, quetógnatos, salpas e doliolídeos). Cada fração foi concentrada em rede de malha $45 \mu \mathrm{m}$ e lavada com água destilada para retirada

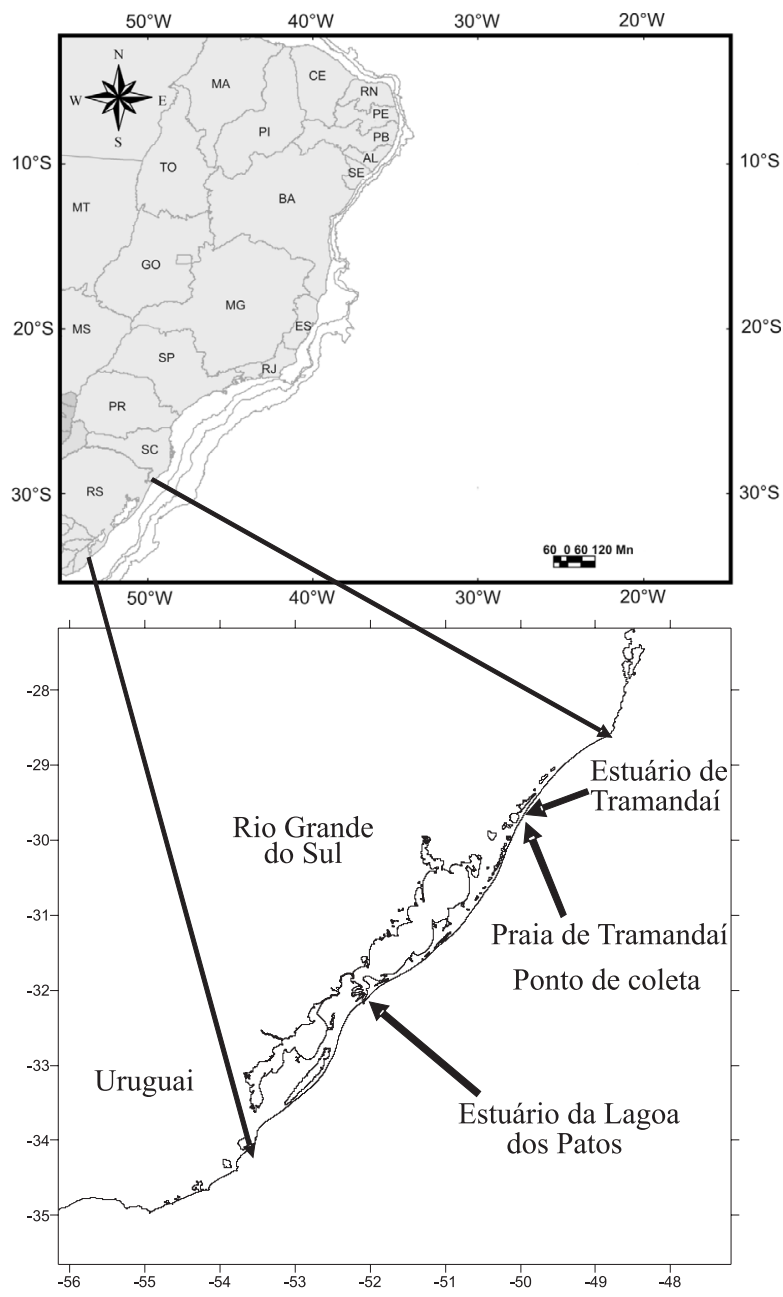

Fig. 1. Mapa do litoral do Rio Grande do Sul, indicando o estuário de Tramandaí, a Praia de Tramandaí e o estuário da Lagoa dos Patos. 
dos sais dissolvidos e em seguida retida em micro-filtro de $0,47 \mu \mathrm{m}$ (GF 52-C) previamente pesados. O material foi levado à estufa a $60^{\circ} \mathrm{C}(\mathrm{BEERs}, 1976)$ por 20 horas. Após este período, os filtros permaneceram durante 6 horas em um dessecador à temperatura ambiente e o material foi pesado em uma balança de cinco casas para a obtenção do peso seco (BERSANO, 1994).

Análise dos dados. Densidade de organismos (org. $\mathrm{m}^{-3}$ ) e índice de diversidade de Shannon (bits.ind ${ }^{-1}$ ) e equitabilidade $(J)$ (PIELOU, 1997) foram calculados para cada unidade amostral para o grupo Copepoda. A freqüência de ocorrência e abundância relativa foram determinadas considerando somente as espécies de copépodes, sendo abundantes aquelas que apresentaram densidade média maior que a densidade média total (LoBo \& LeIGHTON, 1986). Correlações entre variáveis bióticas (densidade de cada taxa em cada coleta ou densidade de cada espécie de copépode em cada coleta) e abióticas (temperatura da água, direção do vento, velocidade do vento, direção da corrente e salinidade) foram avaliadas através da Análise de Correspondência Canônica (ACC). A ACC foi realizada com diferentes combinações de taxóns nas variáveis dependentes (somente Crustacea, somente Crustacea abundantes, somente Copepoda, somente Copepoda abundantes ou somente as espécies indicadoras) com as variáveis independentes (clorofila$a$ e peso seco de zooplâncton foram utilizados ou suprimidos nas diferentes combinações). Um teste de espécies indicadoras (DUfRENE \& LEGENDRE, 1997) utilizando as densidades de organismos foi aplicado agrupando as unidades amostrais de acordo com as estações do ano na tentativa de identificar as espécies características de cada estação. O programa PC-ORD, Versão 4.10 (McCunE \& MEFFORD, 1999) foi utilizado para realizar as análises estatísticas.

\section{RESULTADOS}

Variáveis ambientais. Durante o período de um ano de coleta, a temperatura da água mostrou um padrão de variação sazonal apresentando média de $18^{\circ} \mathrm{C}$ com mínimo de $13^{\circ} \mathrm{C}$ em setembro e novembro de 2005 e máximo de $25^{\circ} \mathrm{C} \mathrm{em}$ janeiro e fevereiro de 2006 (Tab. I). A salinidade esteve acima de 30 em mais de $50 \%$ das amostragens, variando entre 37 em maio e julho de 2006 e 23 em janeiro do mesmo ano. A direção do vento predominante foi nordeste ( $46 \%$ e maioria dos registros feitos na primavera e verão), enquanto os ventos de sudoeste representaram $21 \%$ da freqüência, com principais registros no inverno. A velocidade média do vento foi $3,5 \mathrm{~m} \cdot \mathrm{s}^{-1}$, o valor mínimo foi $0,01 \mathrm{~m} . \mathrm{s}^{-1}$ e máximo de $7,5 \mathrm{~m} . \mathrm{s}^{-1}$ com direção noroeste. A corrente de deriva se descolou em direção sul em 54,16\% das amostragens e em direção norte $45,84 \%$ (Tab. I).

O valor médio de clorofila- $a$ encontrado foi 38,28 $\mu \mathrm{g} . \mathrm{l}^{-1}$, com picos acima de $100 \mu \mathrm{g} . \mathrm{l}^{-1} \mathrm{em}$ maio e junho de 2006 e atingindo $138 \mu \mathrm{g} .1^{-1}$ sob condições de vento sudoeste, em 28 de junho durante um elevado crescimento da microalga Asterionellopsis sp. (Fig. 2). Em janeiro de 2006 foram detectadas altas concentrações de diatomáceas cêntricas e do dinoflagelado Noctiluca, apresentando $90 \mu \mathrm{g} . \mathrm{l}^{-1} \mathrm{de}$ clorofila- $a$. Em dezembro de 2005 foram registrados os menores valores de clorofila- $a\left(4,21 \mu \mathrm{g} . \mathrm{l}^{-1}\right)$ (Fig. 2).

A análise de correspondência canônica não apresentou correlação entre os dados abióticos e a composição, densidade e freqüência das espécies $(p>0,05)$.
Zooplâncton. Um total de 81 táxons foram registrados para o zooplâncton da Praia de Tramandaí. Sua descrição encontra-se em diferentes níveis de identificação (Tab. II). O holoplâncton foi composto por 67 táxons, o meroplâncton por 11 estádios larvais e 3 taxa corresponderam a moluscos e isópodos bentônicos que podem aparecer na coluna d'água devido a ressuspensão causada pela ação de ondas. $\mathrm{Na}$ análise de densidade média dos grupos nas 24 amostras, Mysidacea e Copepoda apresentaram os maiores valores, com 344 e 233 org. $\mathrm{m}^{-3}$ respectivamente, enquanto os outros grupos apresentaram densidades abaixo de 40 org. $\mathrm{m}^{-3}$ (Fig.3).

Copepoda. A Subclasse Copepoda foi dominante entre os grupos identificados com 43 táxons, dos quais 28 pertenceram à Ordem Calanoida, 4 à Ordem Cyclopoida, 3 à Ordem Harpacticoida e 8 à Ordem Poecilostomatoida (Tab. II). A densidade máxima registrada do grupo de copépodes foi de 1884 org.m³ no mês de agosto de 2005

Tabela I. Dados ambientais (T $\left({ }^{\circ} \mathrm{C}\right)$, temperatura da água; Sal,

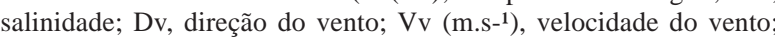
Dc, direção da corrente de deriva) de amostragem quinzenal entre agosto de 2005 e agosto de 2006 na Praia de Tramandaí, RS.

\begin{tabular}{|c|c|c|c|c|c|}
\hline Coletas & $\mathrm{T}\left({ }^{\circ} \mathrm{C}\right)$ & Sal & $\mathrm{Dv}$ & $\mathrm{Vv}\left(\mathrm{ms}^{-1}\right)$ & Dc \\
\hline $23 / 08 / 2005$ & 17 & 32 & $\mathrm{NE}$ & 4,76 & $\mathrm{~N}$ \\
\hline $13 / 09 / 2005$ & 13 & 25 & $\mathrm{SO}$ & 5,03 & $\mathrm{~N}$ \\
\hline $21 / 09 / 2005$ & 15 & 26 & $\mathrm{NE}$ & 3,91 & $\mathrm{~S}$ \\
\hline $04 / 10 / 2005$ & 18 & 30 & $\mathrm{NE}$ & 6,03 & $\mathrm{~N}$ \\
\hline $18 / 10 / 2005$ & 20 & 30 & SO & 3,75 & $\mathrm{~S}$ \\
\hline $01 / 11 / 2005$ & 18 & 30 & $\mathrm{SO}$ & 0,60 & $\mathrm{~S}$ \\
\hline $16 / 11 / 2005$ & 21 & 31 & SE & 2,46 & $\mathrm{~S}$ \\
\hline $29 / 11 / 2005$ & 13 & 33 & $\mathrm{NE}$ & 2,60 & $\mathrm{~N}$ \\
\hline $13 / 12 / 2005$ & 21 & 38 & $\mathrm{NE}$ & 3,75 & $\mathrm{~N}$ \\
\hline $26 / 12 / 2005$ & 16 & 36 & $\mathrm{NE}$ & 3,22 & $\mathrm{~S}$ \\
\hline $10 / 01 / 2006$ & 25 & 24 & $\mathrm{NE}$ & 5,16 & $\mathrm{~S}$ \\
\hline $24 / 01 / 2006$ & 23 & 23 & $\mathrm{NE}$ & 5,65 & $\mathrm{~S}$ \\
\hline $21 / 02 / 2006$ & 25 & 29 & $\mathrm{~S}$ & 2,21 & $\mathrm{~N}$ \\
\hline $07 / 03 / 2006$ & 24 & 36 & & 0,01 & $\mathrm{~S}$ \\
\hline $21 / 03 / 2006$ & 15 & 26 & $\mathrm{~N}$ & 4,93 & $\mathrm{~S}$ \\
\hline $17 / 04 / 2006$ & 19 & 36 & $\mathrm{O}$ & 4,07 & $\mathrm{~N}$ \\
\hline $02 / 05 / 2006$ & 16 & 37 & $\mathrm{NO}$ & 7,47 & $\mathrm{~N}$ \\
\hline $16 / 05 / 2006$ & 18 & 36 & $\mathrm{NE}$ & 4,26 & $\mathrm{~S}$ \\
\hline $30 / 05 / 2006$ & 17 & 34 & & 0,03 & $\mathrm{~N}$ \\
\hline $13 / 06 / 2006$ & 16 & 31 & $\mathrm{NE}$ & 3,48 & $\mathrm{~S}$ \\
\hline $28 / 06 / 2006$ & 15 & 31 & $\mathrm{SO}$ & 1,14 & $\mathrm{~N}$ \\
\hline $13 / 07 / 2006$ & 17 & 34 & $\mathrm{SO}$ & 2,97 & $\mathrm{~S}$ \\
\hline $25 / 07 / 2006$ & 18 & 37 & $\mathrm{NE}$ & 3,13 & $\mathrm{~N}$ \\
\hline $08 / 08 / 2006$ & 17 & 36 & $\mathrm{~N}$ & 3,86 & $\mathrm{~S}$ \\
\hline
\end{tabular}

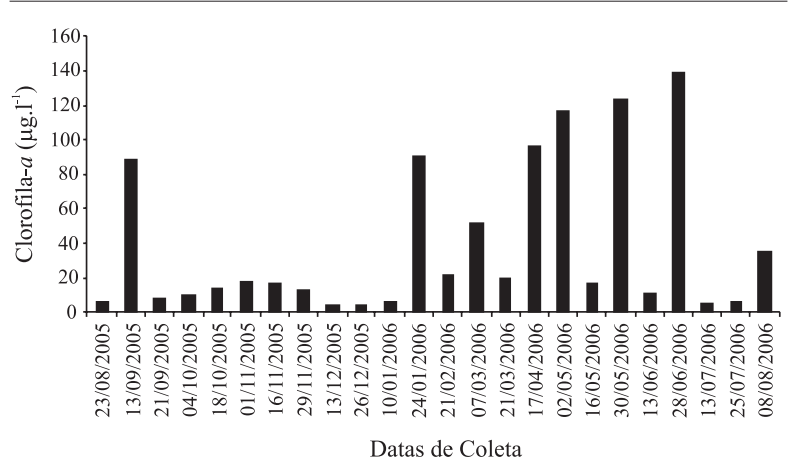

Fig. 2. Variação temporal da concentração de clorofila- $a$ ( $\left.\mu \mathrm{g} .1^{-1}\right)$ na zona de arrebentação da Praia de Tramandaí, RS, no período entre agosto de 2005 e agosto de 2006 
Tabela II. Grupos taxonômicos do zooplâncton identificados na zona de arrebentação da Praia de Tramandaí, RS, entre agosto de 2005 e agosto de 2006.

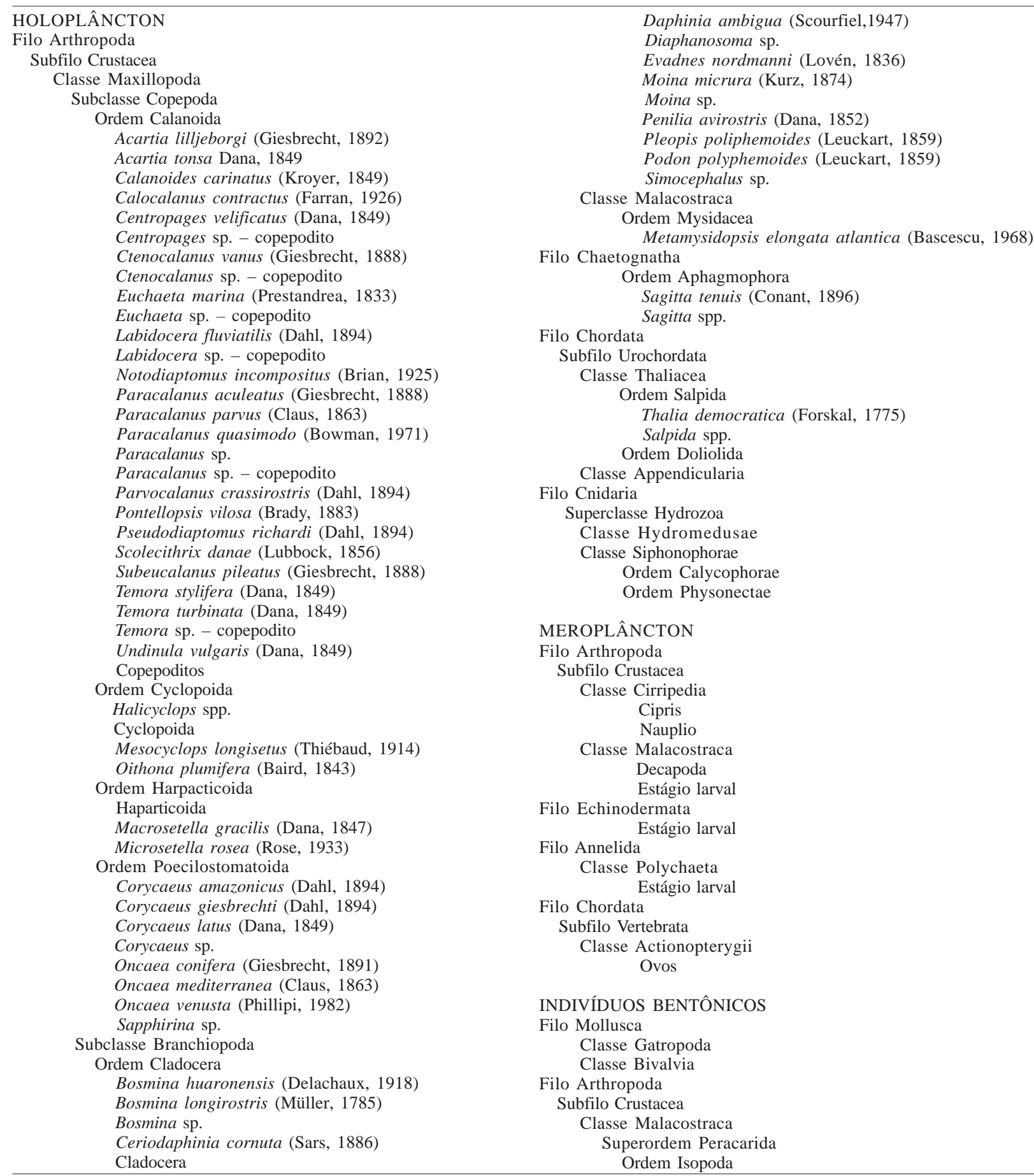

e a riqueza média foi oito espécies com máximo de 17 e mínimo de um. A diversidade ficou em torno de 1,1 bits.ind ${ }^{-1}$ com máximo de 1,8 bits.ind $^{-1}$, em setembro de 2005 (espécies de maior densidade: Ctenocalanus vanus (Giesbrecht, 1888), Labidocera fluviatilis (Dahl, 1894) e Acartia tonsa (Dana, 1849)) e março de 2006 (espécies de maior densidade: Scolecithrix danae (Lubbock, 1856), C. vanus, Temora turbinata (Dana, 1849), Oithona plumifera (Baird, 1843) e Acartia lilljeborgi (Giesbrecht, 1892)) (Fig. 4). O mínimo de zero bits.ind ${ }^{-1}$ foi registrado em maio de 2006, quando somente foi registrado copepoditos de C. vanus (Fig. 4). A equitabilidade variou de zero em novembro de 2005 (quando a riqueza foi de somente uma espécie) a 0,95 em junho de 2006 (Fig. 4).

Do ponto de vista quantitativo os táxons de maior abundância foram os copépodes A. tonsa, Halicyclops spp., C. vanus, Subeucalanus pileatus (Giesbrecht, 1888), T. turbinata, Notodiaptomus incompositus (Brian, 1925) e Paracalanus quasimodo (Bowman, 1971). Temora turbinata foi a espécie que apresentou maior freqüência de ocorrência $(71 \%)$ e abundância relativa (48,96\%), seguida em freqüência por Pseudodiaptomus richardis (Dahl, 1894) 
(63\% e 1,49\%), C. vanus (58\% e $15,91 \%)$, A. tonsa (50\% e 9,52\%) e Parvocalanus crassirostris (Dahl, 1894) (50\% e $0,68 \%)$ (Tab. III).

Acartia tonsa, C. vanus, S. pileatus e T. turbinata foram responsáveis por $79,92 \%$ da abundância relativa de copépodes (Tab. III). Acartia tonsa teve densidade média de 22 org. $\mathrm{m}^{-3} \mathrm{com}$ valores máximos no verão e pico de 271 em fevereiro de 2006, enquanto C. vanus e $T$.

Tabela III. Porcentagem de frequiência observada (FO), densidade média (DM), densidade máxima (Dmáx), densidade mínima (Dmín) e abundância relativa (AR\%) das espécies de copépodes coletadas na zona de arrebentação da Praia de Tramandaí, RS no período de amostragem.

\begin{tabular}{|c|c|c|c|c|c|}
\hline Espécies & $\mathrm{FO}(\%)$ & $\mathrm{DM}$ & Dmáx & Dmín & $\mathrm{AR}(\%)$ \\
\hline \multicolumn{6}{|l|}{ Calanoida } \\
\hline Acartia tonsa & 50,00 & 22,20 & 271,00 & 2,00 & 9,50 \\
\hline Acartia lilljeborgi & 25,00 & 1,80 & 23,00 & 0,17 & 0,80 \\
\hline Calanoides carinatus & 12,50 & 0,14 & 3,10 & 0,03 & 0,05 \\
\hline Calocalanus contractus & 4,70 & 0,13 & 3,00 & 3,00 & 0,05 \\
\hline Centropages sp. copepodito & 4,70 & 0,13 & 3,00 & 3,00 & 0,05 \\
\hline Centropages velificatus & 25,00 & 0,30 & 2,20 & 0,36 & 0,12 \\
\hline Clausocalanus furcatus & 8,30 & 0,14 & 2,00 & 1,47 & 0,06 \\
\hline Copepoditos & 29,70 & 1,30 & 15,60 & 0,35 & 0,54 \\
\hline Ctenocalanus sp. cc & 8,30 & 0,20 & 3,80 & 1,00 & 0,08 \\
\hline Ctenocalanus vanus & 58,30 & 37,00 & 636,00 & 0,70 & 15,91 \\
\hline Euchaeta marina & 4,70 & 0,08 & 1,90 & 1,90 & 0,03 \\
\hline Euchaeta sp. copepodito & 8,30 & 0,08 & 1,40 & 0,66 & 0,04 \\
\hline Labidocera fluviatilis & 29,17 & 0,80 & 12,00 & 0,61 & 0,30 \\
\hline Labidocera sp. copepodito & 16,70 & 0,60 & 10,40 & 1,10 & 0,30 \\
\hline Notodiaptomus incompositus & 25,00 & 7,40 & 93,00 & 1,70 & 3,19 \\
\hline Paracalanus aculeatus & 16,70 & 0,23 & 2,20 & 0,56 & 0,10 \\
\hline Paracalanus parvus & 20,80 & 2,50 & 54,00 & 0,33 & 1,00 \\
\hline Paracalanus quasim & 37,50 & 6,45 & 141,00 & 0,60 & 2,70 \\
\hline Paracalanus sp. & 12,50 & 0,20 & 3,75 & 1,22 & 0,10 \\
\hline Paracalanus sp. cop & 12,50 & 0,60 & 7,13 & 5,19 & 0,30 \\
\hline Parvocalanus crassi & 50,00 & 1,57 & 11,25 & 0,17 & 0,80 \\
\hline Pontellopsis vilosa & 4,20 & 0,04 & 1,00 & 1,00 & 0,02 \\
\hline Pseud & 62,50 & 3,50 & 48,00 & 1,80 & 1,50 \\
\hline Scolecithrix danae & 37,50 & 4,00 & 38,20 & 1,78 & 1,80 \\
\hline Subeucalanus pileat & 45,80 & 13,00 & 74,20 & 1,90 & 5,50 \\
\hline Temora stylifera & 8,30 & 0,20 & 4,00 & 0,80 & 0,09 \\
\hline Temora turbinata & 70,80 & 114,00 & 825,00 & 0,50 & 48,96 \\
\hline Temora sp. copepod & 8,30 & 1,96 & 39,00 & 8,24 & 0,84 \\
\hline Undinula vulgaris & 4,20 & 0,02 & 0,40 & 0,35 & 0,01 \\
\hline \multicolumn{6}{|l|}{ Cyclopoida } \\
\hline Halicyclops spp. & 25,00 & 10,47 & 195,00 & 0,60 & 4,49 \\
\hline Mesocyclops longice & 8,30 & 0,30 & 4,40 & 1,70 & 0,10 \\
\hline Oithona plumifera & 8,30 & 0,53 & 7,40 & 5,45 & 0,20 \\
\hline \multicolumn{6}{|l|}{ Harpacticoida } \\
\hline Harpacticoida $\mathrm{sp}$. & 4,20 & 0,01 & 0,80 & 0,17 & 0,00 \\
\hline la gracilis & 8,30 & 0,08 & 1,30 & 0,66 & 0,04 \\
\hline Microsetella rosea & 4,20 & 0,13 & 3,00 & 3,00 & 0,05 \\
\hline \multicolumn{6}{|l|}{ Poecilostomatoida } \\
\hline Corycaeus sp. & & & ,20 & 1,22 & 0,02 \\
\hline Corycaeus giesbrechti & 8,30 & 0,08 & 1,40 & 0,66 & 0,04 \\
\hline Corycaeus amazonicus & 12,50 & 0,40 & 4,40 & 0,30 & 0,15 \\
\hline Corycaeus latus & 4,20 & 0,05 & 1,30 & 1,30 & 0,02 \\
\hline Oncaea conifera & 4,20 & 0,05 & 1,20 & 1,22 & 0,02 \\
\hline Oncaea mediterra & 4,20 & 0,03 & 0,60 & 0,60 & 0,01 \\
\hline Oncaea venusta & 12,50 & 0,17 & 2,00 & 0,60 & 0,07 \\
\hline Sapphirina sp. & 4,20 & 0,06 & 1,47 & 1,47 & 0,03 \\
\hline
\end{tabular}

turbinata tiveram sua maior densidade em 28 de agosto de 2005 com 636 e 825 org. $\mathrm{m}^{-3}$ respectivamente. Ctenocalanus vanus foi mais freqüente no inverno e $T$. turbinata esteve presente quase o ano inteiro, sendo responsável pelas maiores abundâncias. Subeucalanus pileatus foi registrado em todas as estações com pico de densidade de 74 org. $\mathrm{m}^{-3} \mathrm{em}$ março e julho de 2006, porém com maior freqüência no verão. A figura 5 demonstra a variação de porcentagem representada por cada espécie ao longo do período amostrado.

Outros zooplanctontes. O misidáceo Metamysidopsis elongata atlantica (Bascescu, 1968), foi abundante e freqüente $(58,3 \%)$ na região de Tramandaí apresentando a maior densidade registrada para uma única espécie (3.535 org. $\mathrm{m}^{-3} \mathrm{em}$ 13/09/2005) com média de 320 org. $\mathrm{m}^{-3}$. Cladocera apresentou 14 espécies (4 marinhas e 10 de água doce) (Tab. II) com maior diversidade nos dias 21/ 09/2005 e 10/01/2006, quando também foram registrados copépodes de água doce. Chaetognatha, Salpida, Doliolida, Appendicularia, Actionopterygii na forma de ovos e Siphonophorae estiveram presentes principalmente no verão, com registros de ovos de peixes ainda na primavera e de Chaetognatha nos meses de outono. Entre os principais representantes do meroplâncton foram registrados náuplios e cipris de Cirripedia e larvas de Decapoda (vários estádios de desenvolvimento).

Espécies indicadoras. O teste de espécies indicadoras (DuFRENE \& LEGENDRE, 1997) apresentou A. lilljeborgi,

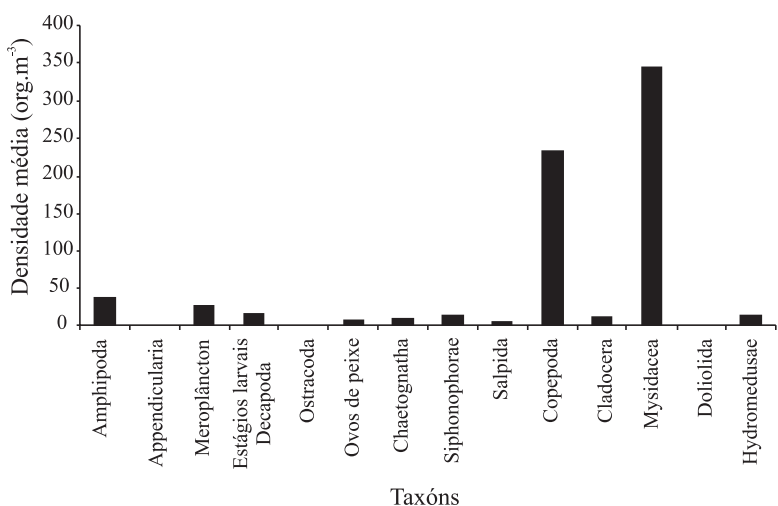

Fig. 3. Densidade média (org. $\mathrm{m}^{-3}$ ) de cada grupo taxonômico registrado na Praia de Tramandaí, RS no período entre agosto de 2005 e agosto de 2006

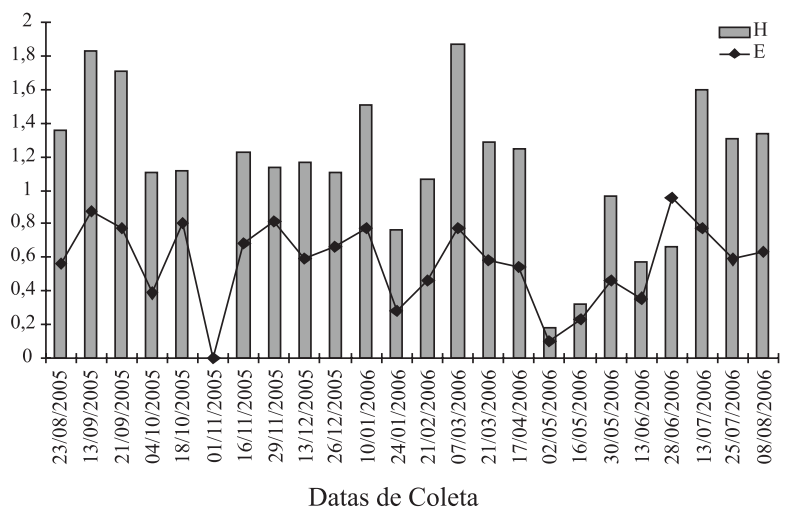

Fig. 4. Variação temporal da diversidade de Shannon $\left(\mathrm{H}-\right.$ bits.ind $\left.^{-1}\right)$ e Equitabilidade (E) dos copépodes identificados na Praia de Tramandaí, RS em cada coleta no período entre agosto de 2005 e agosto de 2006. 
Oithona plumifera, Paracalanus aculeatus (Giesbrecht, 1888), Penilia avirostris (Dana, 1852) e a presença de ovos de peixe como característicos do verão, Corycaeus amazonicus (Dahl, 1894) do inverno e Bathyporeiapus sp. e Bosmina longirostris (Müller, 1785) da primavera, não havendo representantes da estação de outono (Tab. IV).

Biomassa zooplanctônica. Em 13 de setembro de 2005 foi registrado o maior valor de peso seco de zooplâncton total (96,68 mg.m $\mathrm{m}^{-3}$ ) (Fig. 6). Deste total, apenas $0,17 \mathrm{mg} \cdot \mathrm{m}^{-3}$ corresponderam ao grupo de gelatinosos e a grande parte da biomassa foi de crustáceos, nesta amostra majoritariamente representados pela espécie $M$. elongata atlantica (pico de densidade de 3.535 org. $\mathrm{m}^{-3}$ ).

Da mesma forma, ao longo dos meses de coleta a fração de crustáceos das amostras representou de modo geral o maior peso da biomassa. A contribuição dos gelatinosos foi mais freqüiente e significativa durante a primavera e verão e a maior biomassa do grupo foi de $14 \mathrm{mg} . \mathrm{m}^{-3} \mathrm{em}$ março de 2006, apresentando $9 \mathrm{mg} \cdot \mathrm{m}^{-3} \mathrm{e} 7 \mathrm{mg} \cdot \mathrm{m}^{-3} \mathrm{em}$ dezembro de 2005 ejaneiro de 2006 respectivamente (Fig. 6).

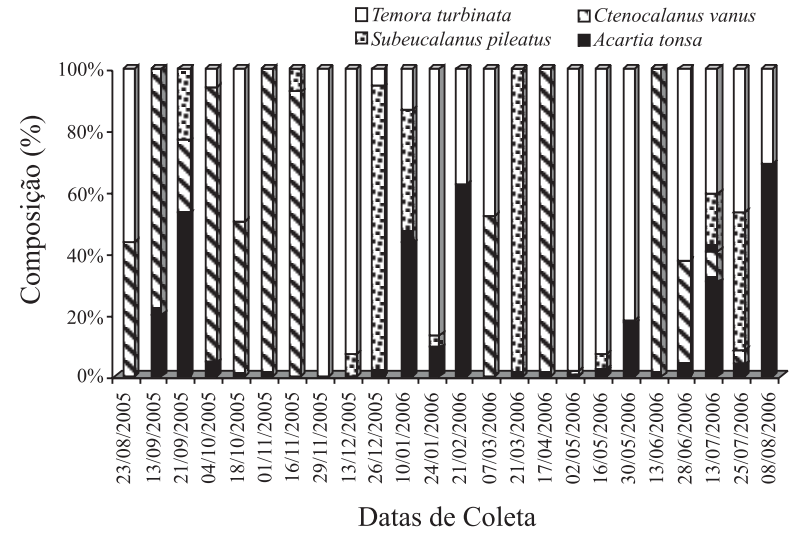

Fig. 5. Variação temporal da porcentagem relativa das espécies mais frequientes e abundantes na Praia de Tramandaí, RS (Temora turbinata (Dana, 1849), Subeucalanus pileatus (Giesbrecht, 1888), Ctenocalanus vanus (Giesbrecht, 1888) e Acartia tonsa (Dana, 1849)) no período entre agosto de 2005 e agosto de 2006

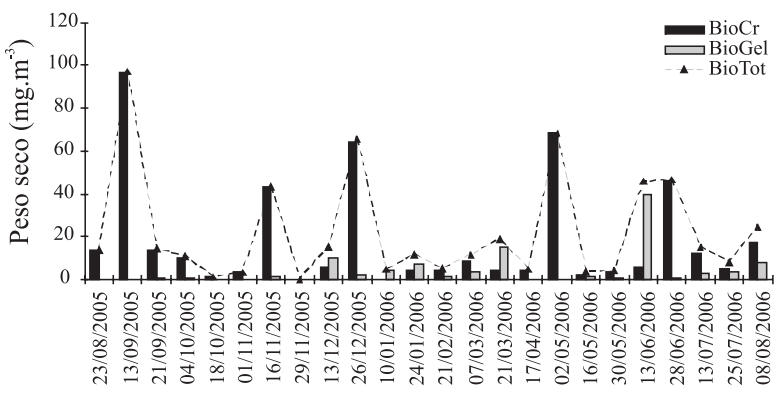

Datas de Coleta

Fig. 6. Biomassa em peso seco $\left(\mathrm{mg} \cdot \mathrm{m}^{-3}\right)$ do zooplâncton de Tramandaí, RS. Biomassa de crustáceos (BioCr), biomassa de gelatinosos (BioGel) e biomassa total (BioTol) no período entre agosto de 2005 e agosto de 2006.

\section{DISCUSSÃO}

Zooplâncton. As variações na composição do zooplâncton observadas ao longo do estudo, reforçam que esta é uma região que recebe contribuições de água de diferentes origens, como a água tropical, de característica mais oligotrófica, transportada em direção sul pela Corrente do Brasil (CAmpos et al., 1995), subantártica, subtropical e central do Atlântico Sul, transportadas para norte pela Corrente das Malvinas (CM) (Piola et al., 1999; CAMPOS et al., 2000) e o aporte de água doce proveniente do deságüe do rio Tramandaí. Assim como em outros estudos sobre o zooplâncton marinho (BJöRNBERG, 1981; BRADFORD-GRIEVE et al., 1999; CAVAlCanti \& LarRaZABál, 2004), o grupo Copepoda foi responsável pela maior diversidade, representado principalmente por espécies da Ordem Calanoida.

Copepoda. Assim como em outras regiões marinhas (Bersano, 1994; SANT' ANNA \& BJÖRNBERG, 2006; SteRZA \& FERNANDEZ, 2006; CoRNILS, et al., 2007), o Grupo Copepoda foi dominante em densidade e riqueza.

A diversidade máxima de Copepoda (1,8 bits.ind $\left.{ }^{-1}\right)$ apresentada em nossos resultados para a zona de arrebentação de Tramandaí é considerada baixa, mas está de acordo com as previsões de MARGalef (1995) e BoltovsCoy (1981), de que a diversidade do plânctoné menor na costa do que nas regiões mais oceânicas e mínimas no estuário. A riqueza de copépodes encontrada (43 taxóns) foi superior a encontrada na zona de arrebentação por BERSANO (1994) (28 taxóns) e próxima à da Baía de Vitória (STERZA \& FERNANDEZ, 2006) (49 taxóns) e Canal de São Sebastião (SANT'ANNA \& BJöRNBERG, 2006) (53 taxóns), também regiões costeiras, ficando bem abaixo dos 110 taxóns de Copepoda registrados em uma região mais afastada da costa no Oceano Índico (Hwang et al., 2006).

A espécie Temora turbinata foi a mais frequente e abundante em nosso estudo. A espécie é considerada de águas oceânicas e costeiras em massas de água tropical e subtropical na margem oeste do Oceano Atlântico Sul (Boltovscoy , 1999). Apesar de hoje ser encontrada no Brasil próximo a estuários em grande abundância (LOPES \& SuÁrez, 1998; Sterza \& Fernandez, 2006), até alguns anos atrás, não havia registro da espécie em estudos realizados no litoral sul do Rio Grande do Sul (Montú, 1980; Bersano,1994). Até meados de 1994, Temora stilyfera (Dana, 1849) era a principal representante do gênero nestas latitudes, sendo T. turbinata encontrada em abundância em águas neotropicais (CHISHOLM \& RoFF, 1990a, b). O primeiro registro da espécie no Brasil foi feito no estuário do rio Vasa-Barris (estado de Sergipe) por ARAúJo \& Montú (1993) e posteriormente por Muxagata \& Gloeden (1995) no estuário da Lagoa dos Patos (RS), sendo considerada espécie invasora destas regiões.

Tabela IV. Espécies do zooplâncton indicadoras $(\mathrm{P}<0,05)$ de cada estação do ano na Praia de Tramandaí, RS, no período entre agosto de 2005 e agosto de 2006.

\begin{tabular}{|c|c|c|c|}
\hline Verão & Outono & Inverno & Primavera \\
\hline Acartia lilljeborgi & & Corycaeus amazonicus & Bathyporeiapus sp. \\
\hline Oithona plumifera & & & Bosmina longirostris \\
\hline Paracalanus aculeatus & & & \\
\hline Penilia avirostris & & & \\
\hline Ovos de peixe & & & \\
\hline
\end{tabular}


Acartia tonsa e Subeucalanus pileatus apresentaram certa freqüência e abundância ao longo do ano na Praia de Tramandaí, da mesma forma que foram freqüentes nas praias do sul do Rio Grande do Sul no levantamento feito por BERSANO (1994) e podem ser encontradas em regiões costeiras e estuários desde a Argentina e ao longo da costa Brasileira (SABATINI \& Martos, 2002; Berasategui et al., 2006; Sterza \& FERNANDEZ, 2006). De acordo com suas distribuições espaciais, A. tonsa foi classificada por Montú (1980) como eurihalina e euritérmica (o que explica sua ampla distribuição latitudinal) porém não atingindo grandes densidades ao se afastar da zona costeira em direção à plataforma aberta. Subeucalanus pileatus é mais resistente a este deslocamento perpendicular à costa.

Neste estudo, Ctenocalanus vanus foi o representante mais importante de águas frias. Esta espécie tem origem subantártica, sendo geralmente dominante em zonas de convergência da Corrente Circumpolar Antártica (BERnARD \& Froneman, 2003, 2005). Na margem oeste do Atlântico Sul, aparece na Argentina com freqüência e grande abundância sobre a plataforma continental (SABATINI \& MARTOS, 2002; BERASATEGUi et al., 2006), sendo registrado no Brasil na região de Cabo frio (VALENTIN et al., 1975; VALENTIN, 1980) em eventos de ressurgência.

Essa espécie pode também chegar à costa riograndense no inverno, sendo transportada pela CM e água costeira acompanhando o fluxo norte do Rio da Prata. Na primavera é possível que sua presença esteja ligada às ressurgências de quebra de plataforma, já que na região próxima a Tramandaí esta é mais estreita se comparada com o litoral sul, permitindo que as águas ascendentes estendam suas influências até mais próximo à costa. Os registros de Calanoides carinatus (Kroyer, 1849) na primavera podem também estar associados aos mesmos eventos.

No trabalho de Berasategui et al. (2006), A. tonsa e $C$. vanus encontram-se espacialmente separados na coluna d'água, com a primeira dominando na região do estuário do Rio da Prata e a segunda na plataforma continental. Nossos resultados não avaliaram a distribuição espacial das espécies, mas demonstram uma tendência à separação temporal com maior freqüência e maior abundância relativa de $C$. vanus no inverno primavera e $A$. tonsa com presença mais significativa no verão, sendo, porém identificada a coexistência entre as espécies.

Notodiaptomus incompositus e Pseudiaptomus richardi são espécies de água doce e estuarinas (MonTú, 1980; Berasategui et al., 2006; SterZa \& FernandeZ, 2006) e foram abundantes e frequientes no ponto de coleta. Segundo Montú (1980), $P$. richardi é mais resistente à variação de salinidade, o que pode explicar sua presença em maior freqüência e abundância na zona de arrebentação de Tramandaí, quando comparado com $N$. incompositus. O registro de ambos copépodes (juntamente com Cladocera) demonstra a forte influência dos deságües para a fauna da região.

Outros zooplanctôntes. O grupo Mysidacea não apresentou grande diversidade de espécies, mas foi o táxon com maior frequiência e abundância registrada durante o período amostral. A importância de misidáceos em zonas de arrebentação também foi ressaltada em trabalhos na zona de arrebentação na África do Sul (McLachlan, 1990; Wooldridge 1983). No Brasil, $M$. elongata atlantica foi registrada no litoral de Santa Catarina (Resgalla, 2001), São Paulo (Lopes et al., 1986; FRIES \& BJÖRNBERG, 1997) e nas praias do sul do Rio Grande do Sul, onde foi reconhecida como a principal responsável pelos valores de densidade e biomassa registrados em Crustacea (Bersano, 1994).

Os grupos Chaetognatha, Salpida, Doliolida, Appendicularia, Hidromedusae e Siphonophorae possuem espécies tanto de águas frias como tropicais (BRAdFord-Grieve et al., 1999), mas neste estudo apresentaram maior freqüência no verão, assim como os ovos de Actionopterygii.

A presença de Cladocera esteve relacionada a salinidades mais baixas, indicando maior influência das águas estuarinas na costa. O estuário de Tramandaí recebe parte do esgoto doméstico das cidades de Imbé e Tramandaí (SoAres, 2002), sendo registrados alguns níveis de contaminação orgânica no mesmo. Outrossim, o registro de Bosmina longirostris e Moina micrura (Kurz, 1874) indicam poluição orgânica na região dos lagos costeiros próximos ao estuário (PEDROzo \& RochA, 2005). Como observado nos resultados, alguns valores de salinidade foram relativamente baixos $(23-26)$, fato que pode estar relacionado com os desagües do rio Tramandaí e com a dinâmica de circulação local. A corrente de deriva litorânea atua diretamente na zona de arrebentação e é gerada pela ação de ondas e ventos, geralmente adquirindo a direção da forçante do vento (McLaChlan, 1980). A ação desta corrente, explicaria os dados de salinidade registrados abaixo da média no ponto de coleta, já que este se localiza ao sul da saída do estuário e sob condições de ventos do quadrante norte pode receber águas menos salinas, transportadas pela deriva litorânea e carreando espécies características de águas doces.

Entre os organismos do meroplâncton, a presença em todas as estações do ano das larvas de Decapoda e os ovos de peixe abundantes durante o verão demonstram a importância da área de estudo como zona de criação. $\mathrm{O}$ Amphipoda Gamaridea do gênero Bathyporeiapus, considerado abundante neste estudo, também é representativo nas praias do sul do Rio Grande do Sul (Bersano, 1994). Assim como observado para a Praia do Cassino (Bersano, 1994), os náuplios e cipris de Cirripédia e também larvas de crustáceo Decapoda foram importantes representantes do meroplâncton.

Biomassa zooplanctônica. Dados elevados de clorofila- $a$ foram freqüientes durante o período de estudo e estiveram geralmente relacionados com a presença de grande concentração de Asterionellopsis sp. ou diatomáceas cêntricas. É interessante notar que os valores mínimos encontrados corresponderam aos mais elevados encontrados por Сіотті et al. (1995) (4.3 mg.m³) na saída do estuário da Lagoa dos Patos. Apesar disto, a biomassa máxima em peso seco foi relativamente baixa quando comparada com o valor máximo de $895,2 \mathrm{mg} \cdot \mathrm{m}^{-3}$ encontrado por BERSANO (1994) na zona de arrebentação da Praia do Cassino, e com as altas concentrações na 
costa da Patagônia Argentina (que chegou a alcançar valores em torno de $1200 \mathrm{mg} \cdot \mathrm{m}^{-3}$ ), com freqüentes valores acima de 300 mg.m. ${ }^{-3}$ (SABATINI et al., 2001).

Espécies indicadoras. Das espécies indicadoras de águas mais quentes, Acartia lilljeborgi, Oithona plumifera e Paracalanus aculeatus representaram a maior influência das massas da Corrente do Brasil nesta estação, já que a primeira é mais abundante do que $A$. tonsa em águas mais quentes (STERZA \& FernANDEZ, 2006) e as outras duas são geralmente abundantes no Nordeste brasileiro (CAVALCANTI \& LARRAZABÁL, 2004). O cladócero Penilia avirostris também classificada aqui como indicadora de verão foi utilizada por VALENTIN et al. (1975) junto com C. vanus, como indicadora de eventos de ressurgência na costa sul do Brasil. Corycaeus amazonicus apareceu somente em coletas de inverno, ao contrário de maior frequiência no verão registrada por BERSANO (1994).

O levantamento da composição, densidade e biomassa do zooplâncton realizado neste trabalho servirá de base para um maior entendimento dos processos de mistura de águas e da capacidade de produção na região da praia de Tramandaí, Rio Grande do Sul.

Agradecimentos. Os autores agradecem ao Centro de Estudos Costeiros Limnológicos e Marinhos (CECLIMAR), em especial ao Osvaldo Stalhban Machado e Manoel Nunes de Melo pelo apoio nas coletas; ao técnico do Laboratório de Zooplâncton da FURG Waldemar José Apolinário Amaral, pela ajuda na análise das amostras; à Epagri/Ciram pelo fornecimento de dados metereológicos da estação de Tramandaí; ao Laboratório de Fitoplâncton da FURG pelo auxílio nas análises de clorofila- $a$ e ao CNPq - Conselho Nacional de Desenvolvimento Tecnológico e Científico pela concessão da bolsa de estudos à primeira autora.

\section{REFERÊNCIAS BIBLIOGRÁFICAS}

Araújo, H. M. P \& Montú, M. 1993. Novo Registro de Temora turbinata (Dana, 1849) (Copepoda, Crustacea) para águas atlânticas. Nauplius 1:89-90.

BeERs, J. R. 1976. Determination of zooplankton biomass. In: SteEdman, H. F. ed. Zooplankton fixation and preservation, Monographs on Oceanographic Methodology. Paris, UNESCO. p.35-86.

Berasategui, A. D.; Marque, S. M.; Gómez-Erache, M.; Ramírez, F. C.; Mianzan, H. W. \& Acha, E. M. 2006. Copepod assemblages in a highly complex hydrographic region. Estuarine, Coastal and Shelf Science 66:483-492.

Bernard, K. S. \& Froneman, P. W. 2003. Mesozooplankton community structure and grazing impact in Polar Frontal Zone of de south Indian Ocean during austral autumm 2002. Polar Biology 26:268-275.

2005. Trophodynamics of selected mesozooplankton in the west-Indian sector of the Polar Frontal Zone, Southern Ocean. Polar Biology 28:594-606.

Bersano, J. G. F. 1994. Zooplâncton da zona de arrebentação de praias arenosas, situadas ao sul de Rio Grande, RS. Primavera de 1990, Verão de 1991. 163p. Dissertação (Mestrado). Fundação Universidade Federal do Rio Grande, Rio Grande. (Não publicada)

BJörnberg, T. S. K. 1981. Copepoda. In: Boltovskoy, D. ed. Atlas del Zooplancton del Atlántico Sudoccidental y métodos de trabajo con el zooplancton marino. Mar del Plata, INIDEP. v.2. p.587-679.

Boltovsкoy, D. 1981. Atlas Del Zooplâncton Del Atlântico Sudoccidental y métodos de trabajo con el zooplancton marino. Mar del Plata, INIDEP. 936p.

1999. South Atlantic Zooplankton. Leinden, Backuys. v.2. p.869-1706

Bradford-Grieve, J. M.; Markhaseva, E. L.; Rocha, C. E. F. \&
Abiahy, B. 1999. Copepoda. In: Boltovskoy, D. ed. South Atlantic Zooplankton. Leiden, Backhuys. v.2. p.869-1098. Brown, A. C. \& McLachlan, A. 1990. Ecology of Sandy Shores. Elsevier. 328p.

Campos, E. J. D.; Gonchlyes, J. E. \& Ikeda, Y. 1995. Water mass characteristics and geostrophic circulation in the South Brazil Bight: Summer of 1991. Journal of Geophysical Research 100 C9:18537-18550.

Campos, E. J. D.; Velhote, D. \& Silveira, I. C. A. 2000. Shelf break upwelling driven by Brazil Current cyclonic meanders Geophysical Research Letters 27(6):751-754.

Cavalcanti, E. A. H. \& Larrazábal, M. E. L. 2004 Macrozooplâncton da Zona Econômica Exclusiva do Nordeste do Brasil (Segunda Expedição Oceanográfica - REVIZEE/NE II) com ênfase em Copepoda (Crustacea). Revista Brasileira de Zoologia 21(3):467-475.

Chisholm, L. A. \& Roff, J. C. 1990a. Abundances, growth rates, and production of tropical neritic copepods off Kingston, Jamaica. Marine Biology 106:79-89.

1990b. Size-weight relationships and biomass of tropical neritic copepods off Kingston, Jamaica. Marine Biology 106:71-77.

Ciotti, A. M.; Odebrecht, C.; Fillmann, G. \& Möller Jr., O.O. 1995. Freshwater outflow and subtropical convergence influence on phytoplankton biomass on the southern Brazilian continental shelf. Continental Shelf Research 15(14): 1737-1756

Cornils, A.; Schnack-Schiel, S. B.; Al-Najuar, T.; Badran, C. M. I.; Rasheed, M.; Manasreh, R.; Richter, C. 2007. The seasonal cycle of the epipelagic mesozooplankton in the northern Gulf of Aqaba (Red Sea). Journal of Marine Systems 68:278-292.

Dufrene, M. \& Legendre, P. 1997. Species assemblages and indicator species: the need for a flexible symmetrical approach PC-ORD, Version 4.10. Ecological Monographs 67:345-366.

Fries, B. G. \& BJöRnBERG, T. K. S. 1997. Aspectos sobre a biologia e ecologia dos misidáceos de São Sebastião, SP, Brasil. In Simpósio de Biologia Marinha, 11º, São Sebastião, 1996. Programa e Resumos... Centro de Biologia Marinha. 30p.

Hwang, J. S.; Souissi, S.; Tseng, L. C.; Seuront, L.; Schmitt, F. G.; Fang, L. S.; Peng, S. H.; Wu, C. H.; Hsiao, S. H.; Twan, W. H.; Wei, T. P.; Kumar, R.; Fang, T. H.; Chen, Q. C.; Wong, C. K. 2006. A 5-year study of the influence of the northeast and southwest monsoons on copepod assemblages in the boundary coastal waters between the East China Sea and the Taiwan Strait. Journal of Plankton Research 28(10):943-958.

LASIAK, T. A. 1986. Juveniles, food and the surf-zone habitat: implications for teleost nursery areas, South Africa Journal of Zoology 21:51-56.

Loвo, E. A. \& Leighton, G. 1986. Estructuras comunitarias de las fitocenosis planctónicas de los sistemas de desembocaduras de rios y esteros de la Zona Central de Chile. Revista Biología Marina 22:1-29.

Lopes, R. M.; Almeida Prado-Por, M. S.; Por, F. D. 1986 Zooplankton seasonality in the Rio Verde estuary (Juréia, São Paulo, Brazil). Revista de Hidrobiologia Tropical 19(34):207-314.

Lopes, S. I. \& Sú́REZ, M. 1998. Copepod assemblage in surface waters of the western gulf of Mexico. Crustaceana 71:312-330.

Margalef, R. 1995. Ecología. Barcelona, Omega. 951p.

McCune, B. \& Mefford, M. J. 1999. PC-ORD. Multivariate Analysis of Ecological Data, Version 4. Oregon, $\mathrm{MjM}$ Software Design. 237p

McLachlan, A. 1980. Exposed sandy beaches as semi-closed ecosystems. Marine Environmental Research 4:59-63. 1990. Surf zone fauna. In: McLachlan, A. \& BRown, A. C. eds Ecology of Sandy Shores. New York, Elsevier. p.165-187.

Montú, A.M. 1980. Zooplâncton do estuário da Lagoa dos Patos I. Estrutura e variações temporais e especiais da comunidade. Atlântica 4:53-72.

Montú, A. M. \& Goeden, I. 1986. Atlas dos Cladocera e Copepoda (crustacean) do Estuário da Lagoa dos Patos (Rio Grande, Brasil). Nerítica 1(2):1-134.

Muxagata, E. \& Gloeden, I. M. 1995. Ocorrência de Temora turbinata Dana, 1849 (Crustacea: Copepoda) no estuário da lagoa dos Patos, RS, Brasil. Nauplius 3:163-164. 
Parsons, T. R.; Matta, Y. \& Lalli, C. M. 1984. A manual of chemical and biological methods for sea water analysis. Oxford, Pergamon. 173p.

Pedrozo, C. S. \& Rocha, O. 2005. Zooplankton and water quality of lakes of the Northern Coast of Rio Grande do Sul State, Brasil. Acta Limnologica Brasileira 17(4):445-464.

Pielou, E. C. 1977. Mathematical ecology. New York, Wiley. $385 \mathrm{p}$.

Piola, A. R.; Campos, E. J. D.; Möller Jr., O. O.; Charo, M. \& Martinez, C. 1999. Continental shelf water masses off eastern South America $-20^{\circ}$ to $40^{\circ} \mathrm{S}$. In : Symposium on Global Changes Studies, $10^{\text {th }}$. Dallas. p.446-449.

ResGalla, JR., C. 2001. Estudo de impacto ambiental sobre a comunidade do Zooplâncton na enseada do saco dos limões, baía sul da ilha de Santa Catarina, Brasil. Atlântica 23:5-16.

Sabatini, M. E.; Gimenez, J. \& Rocco V. 2001. Características del zooplancton del área costera de la plataforma patagónica austral (Argentina). Boletín Instituto Español de Oceanografía 17(3-4):245-254.

Sabatini, M. \& Martos, P. 2002. Mesozooplankton features in a frontal area off northern Patagônia (Argentina) during spring 1995 and 1998. Scientia Marina 66(3):215-232.

SAnT'Anna, E. M. E. \& Bü̈nBerg, T. K. S. 2006. Seasonal dynamics of mesozooplankton in Brazilian coastal waters. Hydrobiologia 563:253-268.

SOARES, I. \& MÖLLER JR., O. 2001. Low-frequency currents and water spatial distribution on the southern Basilian shelf.
Continetal Shelf Reseach 21:1785-1814

SoAres, L. S. 2002. Imbé - Histórico/Turístico. $2^{\mathrm{a} e d .114 p .}$

Souza, R. B.; Mata, M. M.; Garcia, C. A. E.; Kampel, M.; Oliveira, E. N. \& LoREnZzetTI, J. A. 2006. Multi-sensor satellite and in situ measurements of a warm core ocean eddy south of the Brasil-Malvinas Confluence region. Remote and Sensing of Environment 100:52-66.

Sterza, J. M. \& Fernandes, L. L. 2006. Zooplankton community of the Vitória Bay estuarine system (Southeastern Brazil). Characterization during a three-year study. Brazilian Journal of Oceanography 54(2/3):95-105

Tomazelli, L. J. \& Villwock, J. A. 1991. Geologia do Sistema Lagunar Holocênico do Litoral Norte do Rio Grande Do Sul, Brasil. Pesquisas 18(1):13-24.

Valentin, J. 1980. L'écosystème d'upwelling à Cabo Frio (Brésil). Analyse em composantes principales du plâncton à une station fixe. Oceanologia ACTA 3(1):43-50.

Valentin, J.; Macedo, F. E.; Monteiro, W. M. \& Mureb, M. A. 1975. O plâncton na ressurgência de Cabo Frio [Brasil], V - Análise comparativa entre duas estações da Baía de Arraial do Cabo e uma estação fixa oceânica. Período de 04/02 a 16/04/73. Rio de Janeiro, Instituto de Pesquisa da Marinha, Ministério da Marinha. $\mathrm{N}^{\circ} 86: 1-22$

WooldRidge, T. H. 1983. Ecology of beach and surf-zone mysid shrimps in the easthern cape, South Africa, In: McLachlan, A. \& Erasmus, T. eds. Sandy Beaches as Ecosystems. W. Junk, The Hague. p.449-460. 